\title{
Chromosome identification-evolving technology from elucidating condensed chromatin to DNA molecules
}

\author{
A. K. Sharma ${ }^{1}$ - U. C. Lavania ${ }^{2}$
}

Published online: 5 February 2016

(C) Archana Sharma Foundation of Calcutta 2016

The nuclear chromatin which remains dispersed throughout the interphase, resolves itself with the entry of the cell into mitosis or meiosis into definable linear thread-like stainable bodies, called Chromosome. This word "Chromosome" was introduced in a review by German anatomist Heinrich Wilhelm Gottfried Waldeyer [34] has been in use since then because it succinctly defines what early cytologists were able to see with the most modern instrument of their time, a light microscope [38]. With the advancement of knowledge on super-chromosomal organization, it is now known that about $4 \mathrm{~cm}$. long DNA fiber is compacted to form a chromosome $1 \mu \mathrm{m}$ in diameter and $10 \mu \mathrm{m}$ in length [19], with estimated $1 \mu \mathrm{m}$ long continuous string of B DNA equivalent to $3.27 \mathrm{~kb}$ [2]. Further, there is a critical upper limit to chromosome size that a cell can tolerate [27]. At metaphase, the chromosomes are so highly condensed (nearly 10,000 fold) that their morphology can be easily studied under the light microscope. The features presented by mitotic metaphase chromosomes and also the meiotic pachytene offer reliable markers for chromosome identification. The usual form of the nuclear chromosome is the one with localized centromere, in which centromere lies usually at the site of the major (i.e., primary) chromosomal constriction and is the essential marker to classify

\section{U. C. Lavania}

lavaniauc@yahoo.co.in

1 Department of Botany, Centre of Advanced Studies in Cell and Chromosome Research, University of Calcutta, Kolkata 700019 , India

2 Department of Botany, University of Lucknow, Lucknow 226007, India chromosomes on the basis of the sizes of the two arms of a chromosome marked by the position of the centromere.

Lewitsky [13] proposed the term 'Karyotype' to define the phenotypic appearance of a somatic chromosome complement. Levan et al. [12] proposed a classification of chromosomes based on relative ratio between the two chromosome arms differentiated by the position of centromere. In order to elucidate karyotypic differentiation vis-à-vis evolutionary specialization, Stebbins [28] defined the terms symmetric and asymmetric karyotypes. Subsequently, Stebbins [29, 30] provided the classification of karyotypes into 12 categories, ranging from an extreme symmetrical to an asymmetrical karyotype, based on relative proportions of chromosome morphotypes in a given karyotype. Several karyomorphological arrangements elucidating karyotypic details have since been provided [22, 23]. An index called Chromosome "Dispersion Index" depicting statistical gradient of centromere position within the karyotype to facilitate finer differentiation of closely related karyotypes falling within the same "asymmetry" class of Stebbins was developed by Lavania and Srivastava [9], and has been widely used by the cytotaxonomists.

The power of karyotype analysis expanded in late 1960s with the development of banding techniques, including fluorescence and Giemsa staining, reviewed in Lavania [8]. In addition to conventional chromosome markers, morphological differentiation may occur on the basis of the position of centromere (primary constriction), secondary constriction, relative arm ratio. Chromosome banding provide further linear differentiation of chromosomes, facilitating differentiation between the morphologically similar chromosome linkage groups within the karyotype. A new image parameter, codensation pattern (CP), was defined by Fukui and Mukai [3] that has value for identification of the small chromosomes in plants. 


\section{Fluorescence in situ hybridization in chromosome, genome and gene identification}

Whereas, the hybridization of isotope labeled DNA over chromosome in situ provided strong foundation [21], the advent of non-isotopic fluorescent reporter molecules for labeling of DNA in 1980s led to the development of Fluorescence In Situ Hybridization (FISH) techniques [7]. FISH methods have advantage over hybridization with isotope-based probes, including longer probe stability, speed, higher sensitivity, spatial resolution and simultaneous detection of more than one probe. Rayburn and Gill [26] first applied non-isotopic ISH techniques on plant chromosomes using biotin-labelled probes for mapping of repetitive DNA sequences. It is now possible to physically visualize genes and DNA sequences under a microscope on extended DNA fibers prepared from interphase nuclei. The genomic in situ hybridization (GISH) has provided new dimensions to accurately underpin the genome donors in natural polyploids based on direct genomic painting, leading also to detection of artificial hybrids. FISH techniques have since revolutionized cytogenetics in general, and molecular cytogenetics in particular, opening newer opportunities in plant breeding. It is now possible even to discern DNA methylation in situ to visualize epigenetic histone modification marks [11].

\section{Contributions of Yasuhiko Mukai in the development and application of molecular cytogenetic techniques}

Genomic in situ hybridization (GISH) that uses total genomic DNA as probe is one of the excellent technologies suitable for the visualization of whole genome. The classical methods of genome analysis, like pairing of chromosomes at meiosis and pollen fertility of $F_{1}$ hybrids have various limitations, e.g., difficulty in obtaining hybrid plants in trees, effect of $P h$ genes, and so on. The GISH technique has overcome all these problems and successfully discriminated the genomes of many polyploid crops including wheat. Yamamoto and Mukai [35] were the first to apply the FISH technique in wheat.

Yasuhiko Mukai with his group at the Osaka Kyoiku University, Osaka, Japan has developed a leading centre in the development of experimental protocol on finer aspects of chromosome and genome identification with breeding implications [14]. Mukai and Appels [15] were the first to apply in situ polymerase chain reaction (in situ PCR) for mapping plant genes. This method combines the extreme sensitivity of PCR with the cytological location of DNA sequences provided by in situ hybridization. The in situ locations of the rye-specific spacer region were determined on metaphase chromosomes using two pairs of primers designed for rye Nor-Rl and rye 5S-Rrna-R1 sequences. Thus, in situ PCR is expected to be a useful method for amplifying the small region of DNA sequences of specific plant chromosomes and for mapping low copy genes of interest [16].

Physical mapping of low copy sequences Agronomically important genes of wheat are mostly unique or low copy sequences. The information about the exact physical location of agronomically important genes could be useful for a breeding program as well as understanding of the organization of cereal genomes. On human chromosomes, single copy sequences as small as $1 \mathrm{~kb}$ could be routinely detected by the standard FISH technique. But in wheat it is difficult to locate even $10 \mathrm{~kb}$ sequences even when amplification of FISH signals is applied to improve efficiency and realize higher FISH resolution. Large genomic clones such as lambda phages, cosmids, BACs and PACs could be successfully used to realize low copy localization, since large amount of repeated sequences contained in them would facilitate homologous hybridization, signal detection of which would be suppressed by competitive hybridization with unlabelled $C_{0} t-1$ DNA or total genomic DNA. This would enable the unique sequences of interest to be expressively detected. Single or low copy sequences such as genes controlling wheat grain quality have been routinely mapped in wheat chromosomes using lambda phage clones containing insert of 10 to $20 \mathrm{~kb}$ of genomic DNA sequences $[18,24,25]$.

Simultaneous detection of multiple genomes in genomic hybrids Using multicolor FISH, Mukai et al. [17] succeeded in the simultaneous discrimination of the three genomes in hexaploid wheat. Biotin-labeled total genomic DNA of the A-genome progenitor Triticum urartu, digoxigenin-labeled genomic DNA of the D-genome progenitor Aegilops squarrosa, and unlabeled genomic DNA of one of the possible B-genome progenitors, $A$. speltoides, were used as probes and hybridized to chromosome DNA of T. aestivum cv. Chinese Spring. For detection, two fluorochromes, FITCconjugated avidin and rhodamine-conjugated antidigoxigenin, were used. The biotin-labeled probe hybridized to the A-genome chromosomes of wheat detected by yellow fluorescence. The digoxigenin-labeled probe hybridized to the D-genome chromosomes of wheat detected by orange fluorescence. The B-genome chromosome showed faint fluorescence as a result of cross-hybridization of the A- and D-genome probes. Therefore, the A-, B-, and D-genome chromosomes were simultaneously detected by their yellow, brown, and orange fluorescence.

Application of BAC-FISH As bacterial artificial chromosome (BAC) vector can accommodate large-insert genomic DNA, BAC libraries are used most widely at present for analyzing complex plant genomes. Physical mapping by FISH using BAC clones as probes is called BAC-FISH and has been successful in plant species with relatively small genomes, 
such as sorghum, cotton, rice, Arabidopsis, tomato, potato, and wild sweet potato. The large-scale BAC-FISH analysis in the large genome plants, such as wheat and onion, has been studied at conventional molecular cytogenetical level by Mukai's group. To cite a case example, randomly selected 202 BAC clones of Ae. squarrosa (the D-genome donor of hexaploid wheat) were subjected to FISH analysis on chromosomes of common wheat in the absence of $C_{0} t-1$ genomic DNA [31].

FISH analysis on DNA fibers in plants With the aid of FISH techniques, it has now been possible to physically visualize genes and DNA sequences under a microscope on extended DNA fibers (EDFs) from interphase nuclei. In wheat itself EDF fibers could be obtained even from metaphase chromosomes [10]. FISH on extended DNA fibers is a useful tool for determining the sizes of target DNA sequences, the order of genes or clones and their distances in a large chromosomal region $[2,33]$. Currently, fiber FISH has indicated the potential for tracing the target sequences with lengths of up to $2 \mathrm{Mb}$ on single EDFs. This involves a spatial resolution of $1 \mathrm{~kb}$ between adjacent targets, and detection sensitivity of a target of as small as $700 \mathrm{~kb}$ in Triticinae plants [4, 35, 37].

High-resolution mapping of secalin-1 (Sec-1) locus has been performed by FISH to extended DNA fibers of rye, employing DNA probes of lambda phage clones containing the $\omega$-secalin gene $[36,37]$. The fluorescent signals to rye extended DNA fibers revealed continuous strings of $45 \mu \mathrm{m}$, corresponding to the size of $147 \mathrm{~kb}$ DNA. To determine the copy number of $\mathrm{Sec}-1$ locus on DNA fibers, a $1.2 \mathrm{~kb}$ fragment including the entire coding region of the $\omega$-secalin gene and a $1.0 \mathrm{~kb}$ fragment of the promoter region were amplified by PCR as probes for another fiber FISH. Physical position of these sequences was visualized as alternating fluorescent spots by multicolor in situ hybridization. Alternating signals of two DNA probes reflected the tandem repeated organization of Sec-1 locus having 15 copies of the gene. The findings based on Fiber FISH analysis support the contention that the $\omega$ secalin genes are arranged in a head to tail fashion separated by $8 \mathrm{~kb}$ of spacer sequences with a total length of $145 \mathrm{~kb}$ [1]. In transgenic plants, fiber FISH can physically map the transgenes directly on extended DNA fibers and this method is complementary to PCR, Southern blot and sequence analyses $[5,20]$.

Molecular combing has made progress as a new technique to map directly cloned DNA sequences on to individual stretched DNA molecules. Application of molecular combing FISH facilitates determining the structure of clone, copy number of genes, and the order of genes and specific sequences. A small fragment $(2.5 \mathrm{~kb})$ of the starch branching enzyme I gene (Sbe-1) was mapped on DNA molecules of a BAC clone (SBE I) by FISH [32].
Application of FISH to wheat breeding FISH using total genomic DNA as probe i.e., GISH has been shown to be highly sensitive in detecting alien chromatin in cereals, permit the analysis of chromosome and genome arrangements within a nucleus. It allows the determination of the location of translocation breakpoints. GISH has, therefore, become a powerful tool in characterization of alien introgression in wheat [14]. A large number of wheat cultivars have been bred by either gene or chromatin transfer from alien species.

The most important task in current plant breeding research is to introduce a set of agronomically useful genes in a given crop across the barriers of taxonomy and reproductive isolation. It is therefore, necessary in practical plant genomics that suitable transformation systems are established which can facilitate introduction / integration of large genomic fragments loaded with several desired gene functions forming a distant gene source into the recipient crop beyond the barrier of reproductive isolation. FISH could complement such efforts by supplementing information on size of genomic insert and its possible integration sites that would have value in understanding amenability of genomic insertion and its acceptability and expression. This is very important in practical plant breeding since very little is known about where transgenes land, and what effect do they impart onto the recipient organism in terms of stability and expression [14].

\section{Trans-introduction of large wheat genomic fragments into} rice In order to expand genetic variability in rice, Mukai's group has attempted to introduce the large fragments of genomic DNA containing agronomically important genes of wheat into rice. They have successively introduced huge DNA fragments via BAC clones from wheat into rice by 'genome fusion' method. In order to achieve this, as a first step, they developed a system that facilitates efficient introduction of huge mass of wheat genomic DNA into rice. The $75 \mathrm{~kb}$ of Aegilops squarrosa (the D genome donor to common wheat) genome insert containing the wheat isoamylase 1 (TaISA1) gene was transformed to rice by Agrobacterium mediated transformation method using BAC vector [6]. The presence of the transgenes was confirmed in regenerated plants by PCR analysis using primers for a part of the gene. Later, to detect wheat DNA fragments cytologically, FISH experiments were carried out using the original large DNA fragment as a probe in several lines of transgenic rice. Two hybridization signals were observed in metaphase chromosomes in the homozygous $\mathrm{T}_{2}$ plants of each line. Most signals appeared at the terminal or distal regions of rice chromosomes. The fragments of wheat genome DNA were stably transmitted to offspring and the transgenes were expressed in rice [6]. 


\section{In this issue}

The professional associates of Yasuhiko Mukai, an esteemed member of the International Advisory Board of the Nucleus, in a meeting during the International Symposium at Osaka Kyoiku University, Japan in March 2015 desired to contribute scientific articles to felicitate him on completion of 65 years, and express continuity to his professional activities. The 10 articles thus contributed are included in this issue, and a brief account is given below.

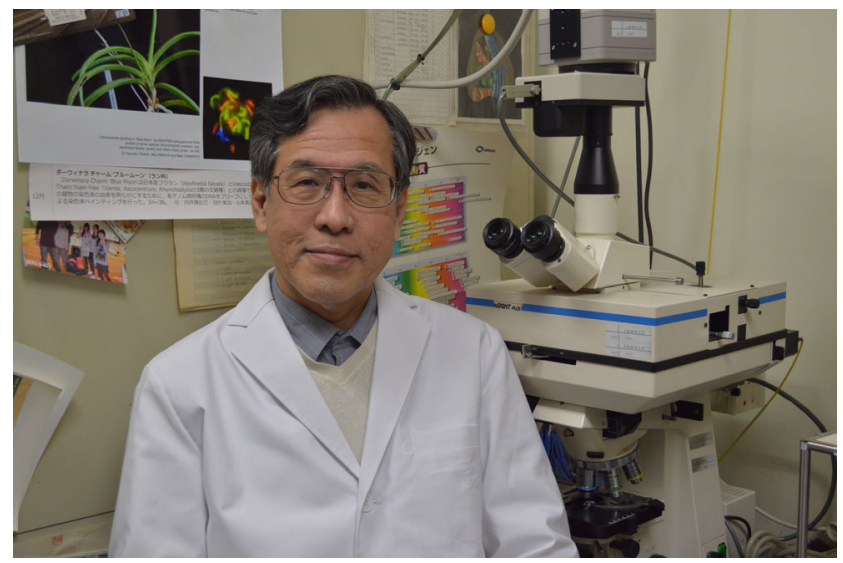

Felicitations to Yasuhiko Mukai

Using Allium hybrid model, Maki Yamamoto (p 165) provides a comprehensive account of significance of cytogenetic and epigenetic factors including functional components of centromere, in regulating the organization of chromosomes in interphase nucleus and meiotic behavior, responsible for sterility / fertility and alternate modes of sustainability.

In addition to cataloguing the available information on conventional and molecular cytogenetics in Orchids, SK Sharma and Y Mukai provide ( $p$ 173) an account of recent developments in the broad scope of epi-cytogenetics in regulating nuclear architecture, spatial-temporal chromosomal distribution of DNA/histone modification marks, and their interplay in the formation of chromatin environment during cell division, and application of flow-cytogenetics in unrevealing the genome architecture and transcriptome and genome sequences in the complex orchid family.

Y Mukai et al. (p 199) using different distant hybrid combinations of wheat allies with Imperata cylindrica have demonstrated that: genome /genotypic specificity plays a key role in haploid induction through Imperata-mediated chromosome elimination process. The $\mathrm{D}$-genome triggers the chromosome elimination and haploid production in wheat $\mathrm{x}$ Imperata. Some D-genome chromosomes (primarily 7D) substituted lines in wheat genetic background may enhance crossability with Imperata in the formation of haploid wheat.
Atsushi Matsuba et al. (p 207) report construction of BAC libraries of two orchid plants, Neofinetia falcata and Rhynchostylis coelestis, as molecular cytogenetic tools, which can be used for chromosome-based comparisons of specific regions between different species and their hybrid chromosomes, suggesting that BAC clones are useful resources for understanding the genomic organization of the orchid plants.

RK Chahota et al. (p 211) provide a case study on FISH based identification of chromosome / chromatin introgression in wheat $\mathrm{x}$ rye derivatives and their association with agronomic characters.

Kanagesswari Muniandy et al. (p 185) have underpinned the significance of molecular cytogenetic tools using BAC clones to visualize the organization of multiple genes citing specific examples of characterization of starch biosynthetic genes in D-genome donor of wheat, Aegilops tauschii.

Anju Shamurailatpam et al. (p 225) using conventional chromosome studies have provided detailed karyomorphological account of wild species of Vigna to underpin prospective utilization of wild genetic resources for genetic enhancement.

Nao Tada et al. (p 217) give an account of mutant breeding of a Japanese traditional black rice cultivar Yayoi-Murasaki to improve seed shattering trait. Pedicel histology has been used to score seed shattering.

Chuan-Ming Yeh and Masaru Ohme-Takagi (p 191) present a comprehensive account of the current understanding of Transcription Factors (TFs) involved in acid stress responses in plants, by describing TFs that are involved in Al toxicity and $\mathrm{P}$ deficient stresses, the two most important stresses in acid soil, and introduce the regulatory networks in response to acid soil stress in plants.

Naohiko Kodo et al. (p 231) provide an account of successful selection of upregulated pleiotropic drug resistance 163 genes in Saccharomyces cerevisiae yrr1-52 GOF mutant cells.

\section{References}

1. Clarke BC, Mukai Y, Appels R. The Sec- 1 locus on the short arm of chromosome 1R of rye (Secale cereale). Chromosoma. 1996;105: 269-75.

2. Fransz PF, Alonso-Blanco C, Liharska TB, Peeters AJM, Zabel P, de Jong JH. High resolution physical mapping in Arabidopsis thaliana and tomato by fluorescence in situ hybridization to extended DNA fibers. Plant J. 1996;9:421-30.

3. Fukui K, Mukai Y. Condensation pattern as a new image parameter for identification of small chromosomes in plants. Jpn J Genet. 1988;63:359-66.

4. Fukui K, Suzuki G, Lagudah ES, Rahman R, Appels R, Yamamoto $\mathrm{M}$, et al. Physical arrangement of retrotransposon-related repeats in centromeric regions of wheat. Plant Cell Physiol. 2001;42:189-96. 
5. Jackson SA, Zhang P, Chen WP, Philips RL, Friebe B, Muthukrishnan S, et al. High resolution structural analysis of biolistic transgene integration into the genome of wheat. Theor Appl Genet. 2001;103:56-62.

6. Kubo A, Rahman S, Utsumi Y, Li Z, Mukai Y, Yamamoto M, et al. Complementation of sugary-1 phenotype in rice endosperm with the wheat isoamylase1 gene supports a direct role for isoamylase1 in amylopectin biosynthesis. Plant Physiol. 2005;137:43-56.

7. Langer-Safer P, Levine M, Ward D. Immunological method for mapping genes on Drosophila polytene chromosomes. Proc Natl Acad Sci U S A. 1982;79:4381-5.

8. Lavania UC. Differential staining and plant chromosomes - a progress in cytogenetics. Curr Sci. 1978;47:255-60.

9. Lavania UC, Srivastava S. A simple parameter of dispersion index that serves as an adjunct to karyotype asymmetry. J Biosci. 1992;17:179-82.

10. Lavania UC, Yamamoto M, Mukai Y. Extended chromatin and DNA fibers from active plant nuclei for high resolution FISH. J Histochem Cytochem. 2003;51:1249-53.

11. Lavania UC, Srivastava S, Lavania S, Basu S, Misra NK, Mukai Y. Autopolyploidy differentially influences body size in plants, but facilitates enhanced accumulation of secondary metabolites, causing increased cytosine methylation. Plant J. 2012;71:539-49.

12. Levan A, Fredga K, Sandberg AA. Nomenclature for centromeric position on chromosomes. Hereditas. 1964;52:201-20.

13. Lewitsky GA. The 'karyotype' in systematics. Bull Appl Bot Genet Plant Breed. 1931;27:220-40.

14. Mukai Y. Perspectives in molecular cytogenetics of wheat. Wheat Inf Serv. 2006;100:17-30.

15. Mukai Y, Appels R. Direct chromosome mapping of plant genes by in situ polymerase chain reaction (in situ PCR). Chromosom Res. 1996;4:401-4.

16. Mukai Y, Yamamoto M. Application of multicolor fluorescence in situ hybridization to plant genome analysis. In: Gupta PK, editor. Genetics and biotechnology in crop improvement. Meerut: Rastogi Publications; 1998. p. 14-23.

17. Mukai Y, Nakahara Y, Yamamoto M. Simultaneous discrimination of the three genomes in hexaploid wheat by multicolor fluorescence in situ hybridization using total genomic and highly repeated DNA probes. Genome. 1993;36:489-94.

18. Mukai Y, Rahman S, Yamamoto M, Okamoto M, Turner M, Li Z, Morell M, Appels R. Physical mapping of genes controlling wheat grain quality by fluorescence in situ hybridization. Proc 9th Int Wheat Genet Symp Saskatoon. 1998; pp. 218-221.

19. Murray AW. How to compact DNA. Science. 1998;282:425-7.

20. Nakano A, Suzuki G, Yamamoto M, Turnbull K, Rahman S, Mukai Y. Integration and organization of large-insert T-DNA in rice: visualization of rearrangements of large transgenes. Mol Gen Genomics. 2005;273:123-9.

21. Pardue ML, Gall JG. Chromosomal localization of mouse satellite DNA. Science. 1970;168:1356-8.
22. Paszko B. A critical review and a new proposal of karyotype asymmetry indices. Plant Syst Evol. 2006;258:39-48.

23. Peruzzi L, Altınordu F. A proposal for a multivariate quantitative approach to infer karyological relationships among taxa. Comp Cytogenet. 2014;8:337-49.

24. Rahman S, Abrahams S, Abbott D, Mukai Y, Samuel M, Morell $\mathrm{M}$, et al. A complex arrangement of genes at a starch branching enzyme I locus in the D genome donor of wheat. Genome. 1997;40:465-74.

25. Rahman S, Regina A, Li Z, Mukai Y, Yamamoto M, KosarHashemi B, et al. Comparison of starch-branching enzyme genes reveals evolutionary relationships among isoforms: Chracterisation of a gene for starch-branching enzyme IIa from the wheat D genome donor Aegilops tauschii. Plant Physiol. 2001;125:1314-24.

26. Rayburn AL, Gill BS. Use of biotin-labeled probes to map specific DNA sequences on wheat chromosomes. J Hered. 1985;76:78-81.

27. Schubert I, Oud JL. There is an upper limit of chromosome size for normal development of an organism. Cell. 1997;88:515-20.

28. Stebbins GL. Variation and evolution in plants. New York: Columbia University Press; 1950.

29. Stebbins GL. Chromosome variation and evolution. Science. 1966;152:1463-569.

30. Stebbins GL. Chromosomal evolution in higher plants. London: Edward Arnold; 1971.

31. Suzuki G, Mukai Y. Plant BAC libraries as tools for molecular cytogenetics. In: Williams CR, editor. Focus on genome research. New York: Nova; 2004. p. 195-210.

32. Suzuki G, Moriyama M, Fujioka K, Yamamoto M, Subramanyam $\mathrm{NC}, \mathrm{Li} \mathrm{Z}$, et al. The starch branching enzyme I locus from Aegilops tauschii, the donor of the D genome to wheat. Funct Integr Genomics. 2003;3:69-75.

33. Suzuki G, Tanaka S, Yamamoto M, Tomita RN, Kowyama Y, Mukai Y. Visualization of the $S$-locus region in Ipomoea trifida: toward positional cloning of self-incompatibility genes. Chromosom Res. 2004;12:475-81.

34. Waldeyer W. Ueber Karyokinese und ihre Beziehungen zu den Befruchtungsvorgängen. Arch Mikrosk Anat. 1888;32:1-122.

35. Yamamoto M, Mukai Y. Application of fluorescence in situ hybridization to molecular cytogenetics of wheat. Wheat Inf Serv. 1989;69:30-2.

36. Yamamoto M, Mukai Y. High-resolution physical mapping of secalin-1 locus of rye on extended DNA fibers. Cytogenet Genome Res. 2005;109:79-82.

37. Yamamoto M, Mukai Y. High-resolution mapping in wheat and rye by FISH on extended DNA fibers. Proc 9th Int Wheat Genet Symp, Saskatoon. 1998; pp. 12-16.

38. Zacharias H. Key word: chromosome. Chromosom Res. 2001;9: 345-55. 